Original Article

\title{
Surgical Outcomes after Superior Vena Cava Reconstruction with Expanded Polytetrafluoroethylene Grafts
}

\author{
Tomoyuki Nakano, MD, ${ }^{1,2}$ Shunsuke Endo, MD, PhD, ${ }^{1,2}$ Yoshihiko Kanai, MD, ${ }^{2}$ \\ Shinichi Otani, MD, ${ }^{2}$ Hiroyoshi Tsubochi, MD, PhD, ${ }^{1}$ Shinichi Yamamoto, MD, PhD ${ }^{2}$ \\ and Kenji Tetsuka, MD, $\mathrm{PhD}{ }^{2}$
}

\begin{abstract}
Objectives: Graft occlusion is a problem after superior vena cava (SVC) reconstruction for thoracic malignancy. Expanded polytetrafluoroethylene (ePTFE) is considered to be an optimal material for venous reconstruction.

Methods: We reviewed the hospital records of 13 patients who underwent complete resection of thoracic malignancy invading the SVC, including SVC reconstruction with ePTFE grafts. Single bypass grafting was performed in two patients (one right-sided, one left-sided) and double bypasses grafting was performed in the other patients. All patients received antithrombotic therapy after surgery. Eight patients died of recurrence or other disease during the follow-up period (range 5-41 months).

Results: Of the 24 grafts in 13 patients, graft patency was confirmed in 20 grafts in 9 patients at a mean time follow-up time of $\mathbf{4 7 . 8} \pm \mathbf{5 0 . 0}$ months after surgery. In the remaining four grafts in four patients, occlusion was diagnosed at a mean time of $1.25 \pm 0.50$ months after surgery. All obstructed grafts were left-sided bypass grafts in patients who underwent double bypass grafting, and did not result in SVC syndrome.

Conclusions: SVC reconstruction with ringed ePTFE grafts was safe and had good outcomes. In patients who underwent double bypasses grafting, the left-sided bypass grafts were susceptible to occlusion.
\end{abstract}

Keywords: superior vena cava (SVC), SVC reconstruction, SVC syndrome, thoracic neoplasm

\section{Introduction}

Mediastinal tumors and lung cancers occasionally

\footnotetext{
${ }^{1}$ Department of General Thoracic Surgery, Jichi Medical University Saitama Medical Center, Omiya, Saitama, Japan

${ }^{2}$ Department of General Thoracic Surgery, Jichi Medical University, Shimotsuke, Tochigi, Japan
}

Received: March 20, 2013; Accepted: April 9, 2013

Corresponding author: Tomoyuki Nakano. Department of General Thoracic Surgery and Cardiovascular Surgery, Jichi Medical University Saitama Medical Center, 847-1 Amanuma-cho, Omiya, Saitama 330-8503, Japan

Email: tcvnknt@jichi.ac.jp

(C)2014 The Editorial Committee of Annals of Thoracic and Cardiovascular Surgery. All rights reserved. invade the superior vena cava (SVC). Such invasion causes SVC syndrome, which reduces quality of life and interferes with the administration of chemotherapy. Treatment of SVC invasion requires a resection of the tumor and SVC reconstruction. However, graft reconstruction of the SVC may result in a low blood flow velocity, leading to obstruction and recurrence of SVC syndrome. Expanded polytetrafluoroethylene (ePTFE) is considered to be an optimal material because of their excellent anti-thrombogenic properties. This report reviews patients who underwent SVC reconstruction surgery with ePTFE grafts at our institute in terms of surgical procedures, graft patency, and clinical course. 
Table 1 Patient characteristics, surgical procedures, graft patency, and clinical course

\begin{tabular}{|c|c|c|c|}
\hline \multirow{2}{*}{$\begin{array}{c}\text { Characteristics } \\
\text { Age (years) }\end{array}$} & \multicolumn{2}{|r|}{ Characteristics } & \multirow[b]{2}{*}{11} \\
\hline & $\begin{array}{l}\text { mean } 46.2 \pm 16.3 \\
\quad \text { range } 23-74\end{array}$ & $\begin{array}{l}\text { Reconstruction procedure } \\
\text { Double bypass }\end{array}$ & \\
\hline Gender & & Right-sided single bypass & 1 \\
\hline Male & 7 & Left-sided single bypass & 1 \\
\hline Female & 6 & Graft diameter & \\
\hline SVC syndrome & $9 / 13(69.2 \%)$ & Right & \\
\hline Histology & & $12 \mathrm{~mm}$ & 4 \\
\hline Thymic neoplasm & 10 & $10 \mathrm{~mm}$ & 8 \\
\hline Thymoma & 2 & Left & \\
\hline Thymic cancer & 2 & $10 \mathrm{~mm}$ & 10 \\
\hline Thymic carcinoid tumor & 2 & $8 \mathrm{~mm}$ & 2 \\
\hline Seminoma & 2 & Intraoperative heparin injection & $12 / 13(92.3 \%)$ \\
\hline Malignant lymphoma & 1 & Insertion method & $11 / 24(45.8 \%)$ \\
\hline Germ cell tumor & 1 & Right-sided bypass & 6 \\
\hline $\begin{array}{l}\text { Lung cancer with lymph node } \\
\text { involvement }\end{array}$ & 3 & Left-sided bypass & 5 \\
\hline Induction therapy & $6 / 13(46.2 \%)$ & Antithrombotic therapy & $13 / 13(100 \%)$ \\
\hline Chemotherapy & 3 & $\mathrm{Wa}+\mathrm{Di}$ & 5 \\
\hline Radiation therapy & 2 & $\mathrm{Wa}+\mathrm{As}$ & 3 \\
\hline \multirow[t]{2}{*}{ Chemoradiation therapy } & 1 & $\mathrm{Wa}+\mathrm{Ti}$ & 2 \\
\hline & & Wa & 2 \\
\hline Surgical approach & & Di & 1 \\
\hline Median sternotomy & 11 & Adjuvant therapy & $11 / 13(84.6 \%)$ \\
\hline Median sternotomy with & & Chemotherapy & 2 \\
\hline posterolateral thoracotomy & 2 & Radiation therapy & 4 \\
\hline Surgical procedure & & Chemotherapy and radiation & \\
\hline Thymothymectomy & 6 & therapy (sequential or concurrent) & 5 \\
\hline $\begin{array}{l}\text { Thymothymectomy, RU lobe } \\
\text { partial resection }\end{array}$ & 3 & Outcome & \\
\hline Thymothymectomy, RUM & & Alive & 4 \\
\hline lobectomy & 2 & Dead & 8 \\
\hline Thymothymectomy, RU & & Unknown & 1 \\
\hline lobectomy & 1 & Survival time (months) & $5-180$ \\
\hline Thymothymectomy, left & & Graft patency & $20 / 24(83.3 \%)$ \\
\hline pneumonectomy & 1 & Right-sided & $12 / 12(100 \%)$ \\
\hline Operative time (min) & mean $450 \pm 132$ & Left-sided & $8 / 12(66.7 \%)$ \\
\hline Blood loss (g) & $\begin{array}{c}\text { range } 255-605 \\
\text { mean } 2597 \pm 1287, \\
\text { range } 965-4530\end{array}$ & $\begin{array}{l}\text { Duration of patency after surgery } \\
\text { (months) }\end{array}$ & $\begin{array}{l}\text { mean } 47.8 \pm 50.0 \\
\text { range } 1-150\end{array}$ \\
\hline
\end{tabular}

SVC: superior vena cava; RU: right upper; RUM: right upper middle; Wa: warfarin; Di: dipyridamole; Ti: ticlopidine; As: aspirin

\section{Materials and Methods}

The hospital records of the 13 patients with mediastinal tumors or lung cancers invading the SVC who underwent surgical resection with SVC reconstruction from February 1991 to December 2011 were reviewed (Table 1). The patients were seven males and six females, with a mean age of $46.2 \pm 16.3$ years. Ten patients had thymic tumors, including two with thymoma, two with thymic cancer, two with thymic carcinoid tumor, two with seminoma, one with mediastinal metastasis of a germ cell tumor (embryonal cell carcinoma), and one with malignant lymphoma. Three patients had primary lung cancer with lymph node involvement. Nine patients had SVC syndrome before undergoing treatment. Induction therapy was performed in six patients, including chemotherapy in three patients, radiation therapy in two patients, and chemoradiation therapy in one patient. The patient characteristics are shown in Table $\mathbf{1 .}$ 


\section{Surgical Technique}

Surgery was performed via median sternotomy in 11 patients, and via median sternotomy plus lateral thoracotomy in 2 patients. The SVC and brachiocephalic veins $(\mathrm{BCV})$ were reconstructed with ringed ePTFE grafts in all patients. Double bypasses grafting was performed in 11 patients. In patients who underwent double bypasses grafting, an $8-10 \mathrm{~mm}$ diameter left-sided bypass graft was placed between the left BCV and the right atrial appendage. The trabeculae of the right atrial appendage were trimmed before anastomosis. After en bloc tumor resection, a 10-12 mm diameter right-sided bypass graft was placed between the right $\mathrm{BCV}$ and the SVC graft. Anastomoses between the grafts and the vessels or atrial appendage were performed using a continuous polypropylene suture, or by insertion of the graft into the vessel or atrial appendage followed by fixing with sutures. Insertion method was chosen for the patients with an anastomosis expected to bend and/or twist. In 12 patients, the activated coagulation time was adjusted to $200 \mathrm{~s}$ during SVC reconstruction by administration of intravenous heparin. En bloc tumor resection including the SVC was performed with a single bypass graft in two patients (one left-sided, one right-sided). In the patient who underwent single left-sided bypass grafting, it was not possible to perform the proximal anastomosis needed for right-sided bypass grafting because the tumor had invaded into the proximal SVC (Fig. 1). In the patient who underwent single right-sided bypass grafting, one end of the graft was anastomosed end-to-end with the right $\mathrm{BCV}$, followed by insertion of the graft into the SVC during a short period of clamping.

The operative time ranged from 255 to 605 minutes (mean: $450 \pm 132$ minutes) and the blood loss ranged from 965 to $4530 \mathrm{~g}$ (mean: $2597 \mathrm{~g} \pm 1287 \mathrm{~g}$ ). All patients remained hemodynamically stable during the operation. There was no operative mortality. The surgical procedures are shown in Table $\mathbf{1}$.

\section{Postoperative Care and Follow-up}

All patients received antithrombotic therapy after surgery, consisting of one or more of warfarin, aspirin, dipyridamole, and ticlopidine. Graft patency was evaluated by an intravenous digital subtraction angiography or an enhanced computed tomography, 1-3 months after surgery, followed by variable evaluation times among patients. Eleven patients received adjuvant therapy: two

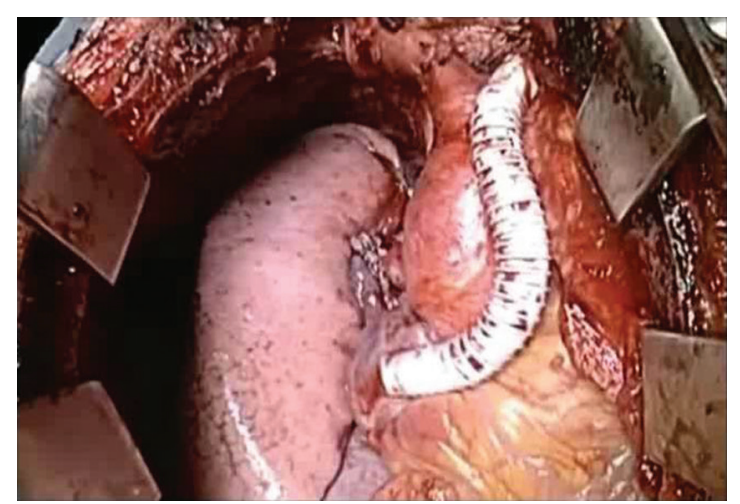

Fig. 1 Operative findings in the patient who underwent single left-sided bypass grafting following thymothymectomy concomitant with right upper and middle lobectomy. The right-sided bypass was not possible because the thymic tumor had invaded into the proximal SVC. A 10-mm diameter ringed ePTFE graft was placed between the left BCV and the right atrial appendage. Note that the peripheral end of the graft was anastomosed to left BCV with no distortion. SVC: superior vena cava; ePTFE: expanded polytetrafluoroethylene; BCV: brachiocephalic veins

received chemotherapy, four received radiation therapy, and five received chemoradiation therapy. The clinical course is shown in Table 1.

\section{Statistical Analysis}

The 24 grafts in 13 patients were divided into two groups: a patent group and an occluded group. Quantitative parameters were compared between the two groups using the $\chi^{2}$ test. A $p$ value of $<0.05$ was considered statistically significant. Statistical analyses were performed using the Statcel 2 software package (OMS Ltd., Saitama, Japan).

\section{Results}

At the end of the follow-up period, eight patients had died of recurrence or other disease, two remained alive with recurrence, two remained alive without recurrence, and it was unknown whether the other patient was still alive. The survival time ranged from 5 to 180 months (Table 2). The overall survival rates were $92.3 \%$ at 1 year, $46.2 \%$ at 3 years, and $38.5 \%$ at 5 years. In patients with thymic tumors, the overall survival rates were $90.0 \%$ at 1 year, $50.0 \%$ at 3 years, and $50.0 \%$ at 5 years (Fig. 2). 
Table 2 Comparisons of clinical factors and grafting methods between patent and occluded grafts

\begin{tabular}{lccc}
\hline Factor & Patent grafts $(\mathrm{n}=20)$ & Occluded grafts $(\mathrm{n}=4)$ & $p$-value \\
\hline Operative time (min) & mean $455 \pm 128$ & mean $517 \pm 89$ & 0.28 \\
Blood loss $(\mathrm{g})$ & mean $2538 \pm 1222$ & mean $3334 \pm 1582$ & 0.40 \\
Reconstruction procedure & & & 0.69 \\
$\quad$ Double bypass & 18 & 4 & \\
$\quad$ Single bypass & 2 & 0 & 0.047 \\
Graft location & & & \\
$\quad$ Left-sided & 8 & 0 & 0.30 \\
$\quad$ Right-sided & 12 & 0 & \\
Graft diameter & 4 & 3 & 0.23 \\
$12 \mathrm{~mm}$ & 15 & 1 & \\
$10 \mathrm{~mm}$ & 1 & 1 & \\
$8 \mathrm{~mm}$ & 12 & 3 & \\
Insertion method & 8 & & \\
No & & & \\
Yes & & & \\
\end{tabular}

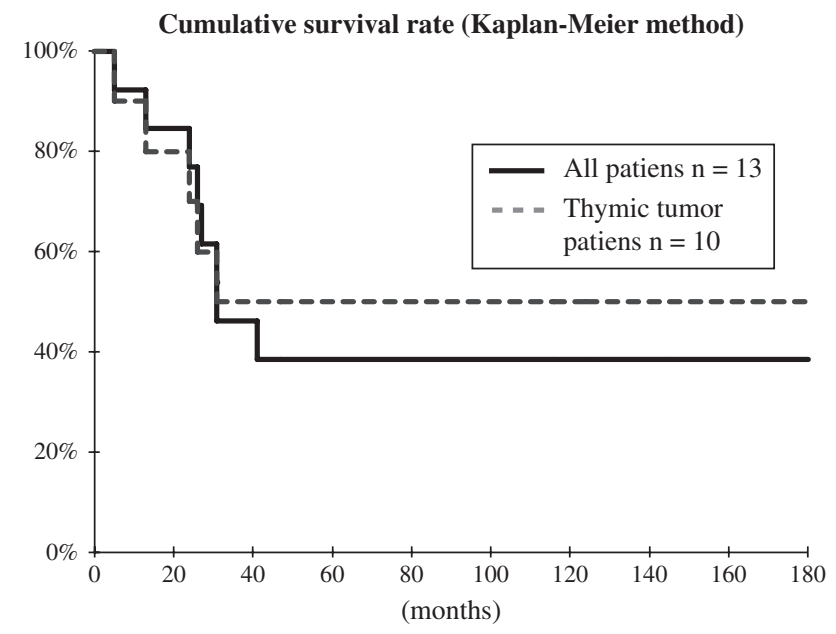

Fig. 2 Cumulative survival rates (Kaplan-Meier method) in all patients $(\mathrm{n}=13)$ who underwent SVC reconstruction, and in patients with thymic tumors $(\mathrm{n}=10)$ who underwent SVC reconstruction.

The graft patency is shown in Table $\mathbf{1}$. Of the 24 grafts in 13 patients, 20 grafts in 9 patients were patent and 4 grafts in 4 patients were occluded at the time of postoperative intravenous digital subtraction angiography or enhanced computed tomography, giving an overall graft patency rate of $83.3 \%$. Graft occlusion was identified at 1-2 months (mean: $1.25 \pm 0.50$ months) after surgery. No additional intervention was required as there were no symptoms related to graft occlusion. Patency was last confirmed in the non-occluded grafts at 1-150 months (mean: $47.8 \pm 50.0$ months) after surgery.

In the patient with early recurrence after bypass surgery, patency was not followed up in the long term. All occluded grafts were left-sided bypass grafts (left $\mathrm{BCV}$ to right atrial appendage) in patients who underwent double bypasses grafting, giving a left-sided graft patency rate of $63.6 \%$ for this procedure. The two patients with single bypass grafting maintained graft patency with no graft complications. Statistical analysis showed that the left-sided bypass graft of patients who underwent double bypasses grafting was significantly more likely to occlude than other grafts (Table 2).

\section{Discussion}

The most frequent symptoms and signs caused by SVC obstruction include cough, headache, nausea, dyspnea, tongue swelling, facial swelling, and upper limb swelling. ${ }^{1)}$ SVC syndrome is disabling, and may have potentially life-threatening complications. ${ }^{2}$ ) Thoracic malignant tumors that involve the great vessels of the mediastinum used to be considered inoperable because of the poor prognosis, challenging surgery, and low graft patency rates. However, en bloc tumor resection and replacement of the SVC may be indicated in selected patients with lung cancer without nodal involvement, or with invasive thymoma. The reported 5-year survival rates after complete en bloc resection including the SVC are $31 \%$ in patients with non-small cell lung cancer and $42 \%$ in patients with thymic tumors. ${ }^{3-5)}$ 


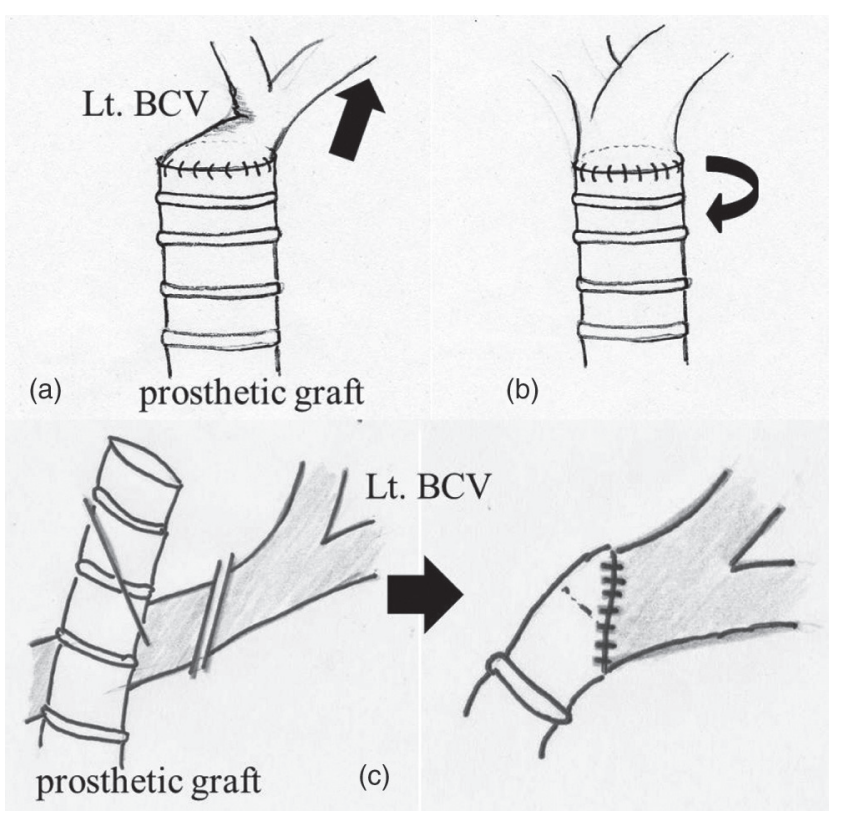

Fig. 3 Schematic representation of an anastomosis between a leftsided prosthetic graft and the left BCV. An anastomosis between the left BCV and a prosthetic graft tends to bend (a) because of the different elasticity of the vessel and a vascular prosthesis and twist (b) because of the different axes of the left BCV and the SVC. The end of the graft should be trimmed according to the length and the three-dimensional configuration of the left BCV $(\mathbf{c})$. The bend and twist of the anastomosis could be prevented by adjusting cutting planes of the graft and the left BCV.

Prosthetic graft complications are associated with a mortality rate of $4.5 \%$ to $12 \%{ }^{6,7)}$ The most dangerous complication is thrombosis with pulmonary embolism. Thrombosis is often related to technical failure of the anastomosis, or the thrombogenic characteristics and diameter of the prosthetic graft. Various biologic and synthetic materials, such as bovine pericardium, Dacron, and PTFE have been used for vessel reconstruction. Bovine jugular vein is associated with high risk of thrombosis. ${ }^{8)}$ In animal experiments, ePTFE grafts were found to have good long-term patency because of their excellent anti-thrombogenic properties when used in the low-pressure venous system. Therefore, ePTFE is now considered to be an optimal material for SVC reconstruction. ${ }^{9)}$ Shimizu, et al. performed SVC reconstruction using ePTFE grafts in six patients with malignant thymic tumors (five SVC grafts and six left BCV grafts), and reported occlusion of one left BCV graft at 1 month after surgery. ${ }^{10)}$
Patency rates may be lower in patients with double bypass grafts than in those with single bypass grafts. In patients with double bypass grafts, Okada, et al. reported graft patency rates of $82 \%$ on the right side and $38 \%$ on the left side. ${ }^{11)}$ In our study, graft patency rates were $100 \%$ on the right side and $66.7 \%$ on the left side. In patients with double bypass grafts, the reasons for left-sided grafts being more susceptible to occlusion are thought to be the smaller diameter of the left BCV compared with the right $\mathrm{BCV}$ and $\mathrm{SVC}$, and compression of the left-sided graft by the manubrium of the sternum after closure of the chest. ${ }^{12)}$ In addition, there may be decreased blood flow in the left-sided graft due to stealing by the right-sided graft. The anastomosis between the left $\mathrm{BCV}$ and the prosthetic graft also tends to twist and bend because the axis of the left BCV is different to the axis of the SVC. The end of the graft should therefore be trimmed according to the three-dimensional configuration of the left BCV (Fig. 3). The trabeculae of the atrial appendage can prevent secure anastomosis, and should be sufficiently trimmed.

\section{Conclusion}

SVC reconstruction with ringed ePTFE grafts was safe and resulted in excellent patency, even with single bypass grafting. Right-sided single bypass grafting could be safely completed during total occlusion of the SVC, with operative time shortened by using an insertion procedure of the graft into the right atrium. Left-sided single bypass grafting should be avoided, due to the low patency rates of left-sided grafts.

\section{Disclosure Statement}

The authors declare that they have no conflict of interest.

\section{References}

1) Porte H, Metois D, Finzi L, et al. Superior vena cava syndrome of malignant origin. Which surgical procedure for which diagnosis? Eur J Cardiothorac Surg 2000; 17: 384-8.

2) Panneton JM, Andrews JC, Hofer JM. Superior vena cava syndrome: relief with a modified saphenojugular bypass graft. J Vasc Surg 2001; 34: 360-3.

3) SpaggiariL, Thomas $P$, MagdeleinatP, etal. Superior vena cava resection with prosthetic replacement for nonsmall cell lung cancer: long-term results of a multicentric 
study. Eur J Cardiothorac Surg 2002; 21: 1080-6.

4) Bacha EA, Chapelier AR, Macchiarini P, et al. Surgery for invasive primary mediastinal tumors. Ann Thorac Surg 1998; 66: 234-9.

5) Borri A, Leo F, Veronesi G, et al. Extended pneumonectomy for non-small cell lung cancer: morbidity, mortality, and long-term results. J Thorac Cardiovasc Surg 2007; 134: 1266-72.

6) Dartevelle PG. Herbert Sloan Lecture. Extended operations for the treatment of lung cancer. Ann Thorac Surg 1997; 63: 12-9.

7) Spaggiari L, Regnard JF, Magdeleinat P, et al. Extended resections for bronchogenic carcinoma invading the superior vena cava system. Ann Thorac Surg 2000; 69: 233-6.

8) Schoof PH, Koch AD, Hazekamp MG, et al. Bovine jugular vein thrombosis in the Fontan circulation. J Thorac Cardiovasc Surg 2002; 124: 1038-40.

9) Fiore AC, Brown JW, Cromartie RS, et al. Prosthetic replacement for the thoracic vena cava: an experimental study. J Thorac Cardiovasc Surg 1982; 84: 560-8.

10) Shimizu N, Date H, Moriyama $S$, et al. Reconstruction of the superior vena cava in patients with mediastinal malignancies. Eur J Cardiovasc Surg 1991; 5: 575-8.

11) Okada M, Sugimoto T, Yamamoto H. [Surgical strategy for invasive pulmonary and mediastinal tumors requiring superior vena cava reconstruction]. Kyobu Geka 1999; 52: 14-8. [in Japanese with English abstract]

12) Okada M, Sugimoto T. [Technical points of venous reconstruction for disorders of the superior vena cava]. Kyobu Geka 2004; 57: 89-94; discussion 95-7. [in Japanese with English abstract] 